\title{
Effect of L-Carnosine, DHA and vitamin D3 on children with down's syndrome- a pilot study on Indian population
}

\author{
Chandrasekhar P. ${ }^{1}$, Ramachandran S. ${ }^{2}$ \\ ${ }^{1}$ Dr. Priya Chandrasekhar, Pediatrician, Ramana Maharishi Rangammal Hospital, Thiruvannamalai, Tamil Nadu, India, \\ ${ }^{2}$ Dr. Surekha Ramachandran, Phd., President, Down's syndrome federation of India, Chennai, Tamil Nadu, India.
}

Correspondence Author: Dr. Priya Chandrasekhar, E-mail Id: doctorpriyac@gmail.com

\begin{abstract}
Background: Down syndrome (DS) is one of the leading genetic causes of intellectual disability (ID) accounting 15$20 \%$ of ID population across the world. Early intervention is the potential treatment; alternative therapies like occupational and physical therapy are carried to improve different skills in the child with DS. L-Carnosine is the first dietary supplement that helps in improving cognitive and behavioural symptoms. Docosahexaenoic acid (DHA) improves learning and memory abilities. Vitamin D3 is a therapeutic neuroprotector and its supplementation with DHA improves certain behaviours associated with ADHD, bipolar disorder, schizophrenia, and impulsive behaviour by regulating serotonin production and function. This study was aimed to determine the synergetic effect of L-Carnosine, DHA and Vitamin D3 in improving development in children with Down syndrome. Materials and Methods: The basic design of the study was an open label, placebo-controlled study with study duration of six months with 60 patients and analysis were carried out with the questionnaire created in reference to Ages and Stages Questionnaire (ASQ). The main parameters studied were communication, gross motor, fine motor, problem solving, social and vocabulary skills. Results: Our results confirm that parameters related to communication, fine motor, gross motor, social skills, problem solving and vocabulary significantly improved in children who were supplemented with additional nutritional supplementation, when compared to the children who were notand the data were statistically significant $p<0.05$. Conclusion:The combination of L Carnosine, DHA and Vitamin D3 supplementation yields an increase in cognitive processing speed, language skills and improved motor skills.
\end{abstract}

Key words: Down syndrome, L Carnosine, Docosahexaenoic acid, Vitamin D3

\section{Introduction}

Down syndrome (DS) is one of the leading genetic causes of intellectual disability (ID) in the world. DS alone accounts for $15-20 \%$ of ID population across the world. This condition arises from certain types of disturbance in genetic mechanism that leads to the development of an extra chromosome [1]. DS are classified into three main categories. Trisomy, which is the most common type and accounts for $95 \%$ of the total DS population.

Translocation and mosaic are less prevalent; these accounts for $3 \%$ and $2 \%$, respectively [2]. People with DS are susceptible to various chronic disorders, infections, and disabilities. In India, various organizations are primarily involved in the treatment of conditions present in children with Down syndrome.

Manuscript received: $27^{\text {th }}$ March 2018

Reviewed: $4^{\text {th }}$ April 2018

Author Corrected: $10^{\text {th }}$ April 2018

Accepted for Publication: $14^{\text {th }}$ April 2018
Various therapies are suggested upon early intervention, normal activities such as dressing, grooming are as occupational therapy, other therapies such as physical therapy improves motor skills, speech therapy and language therapy [1,2]. Advancements in Medical Technologies have improved the quality of life of individuals with Down syndrome (DS).

Individuals with Down syndrome (DS) have high levels of oxidative stress throughout their lifespan[3]. Oxidative stress leads to the metabolism and release of free radicals, the loss of balance created can directly start the neuropathology of DS and a tendency to cause Alzheimer Disease (AD) [4,5].

L-Carnosine: L-Carnosine, an antioxidant exerts both direct and indirect effects that are mediated by different mechanisms involving metal ion chelation, and scavenging reactive oxygen species and peroxyl 
radicals $[6,7]$. It was first noted in the olfactory bulb and nasal olfactory epithelium of rodents at concentrations higher than any other brain region. It is present in the CNS and has been proposed that carnosine is a naturally occurring suppressor of oxidative damage in olfactory neurons [8]. It is the first dietary supplement that may alter the neuronal function in children with this disorder and help in improving cognitive and behavioural symptoms [9].

Carnosine is widely used as a dietary supplement with potential role in treating Alzheimer's disease, autism, cataract prevention, brain ischemia, Parkinson's disease, Down's syndrome, epilepsy, schistosomiasis, and aging [8]. Data suggest that carnosine is an endogenous neuroprotector. It has been argued that the presence of other endogenous antioxidant systems (glutathione, vitamins $\mathrm{C}$ and $\mathrm{E}$ ) availability is very low in the nervous system, which is compensated for by (homo) carnosine. It has been found that Carnosine offers cerebro- protection against histological and functional damage without any toxicity in ischemic models $[10]^{13}$ Carnosine may be effective to control Alzheimer's disease, possibly on account of its ability to suppress the toxic effects of amyloid beta towards cultured cells [11].

Carnosine has a protective or activating role in the frontal lobe. Of note, frontal lobe dysfunction is hypothesized to be linked to expression and behaviour; both areas are impaired in ASD [8]. Studies conducted in humans have shown its benefits in terms of enhancing cognition and well-being. L-carnosine offersgreat potential for the treatment of NDDs and its efficacyasa neuro protective agent, of which antioxidantaction is presumed to be the most beneficial [12].

DHA \& Vitamin D3: Docosa hexaenoic acid (DHA) improves memory and neurogenesis either supplemented separately or in combination with phosphorlipids uridine [13-15]. Animal studies has shown that DHA supplementation improves the production of brain-derived neurotrophic factor (BDNF), a neurogenesis mediator [16]. Taken together, research results suggest that DHA, vitamin D and uridine are of importance for optimal brain function. Brain development is rapid in the early years of life and hence it is more sensitive to deficiencies of vital nutrients [17]. DHA is important for the development of sensory, perceptual, cognitive, and motor neural systems at the time of brain growth spurt [18]. Vitamin D3 and its analogs have the potential for development as therapeutic neuro protectors [19]. Supplementation with vitamin D and DHA improves certain behaviours associated with ADHD, bipolar disorder, schizophrenia, and impulsive behaviour by regulating serotonin production and function [20]. Considering their synergistic action, vitamin D and DHA, supplemented together, can go a long way in optimum neuro development of children in their crucial years of growth [21]. This study aimed to determine the effect of a combination of L-Carnosine, vitamin D3 and DHA supplementation on cognitive abilities in Down's syndrome children

\section{Methods}

Place of Study: This present study was carried out in Ramana Maharishi Rangammal Hospital, Thiruvannamalai, Tamil Nadu and Down's syndrome federation of India, Chennai.

Type of Study: Randomized controlled study

Sampling methods: The patients who met the inclusion criteria and no exclusion criteria were enrolled in the study after due informed consent from the parents/LAR. Totally 60 children participated in the study and randomly assigned in to two groups in the ration 1:1. One arm received L Caronisine (Cognicare ${ }^{\mathrm{TM}}$ ) $200 \mathrm{mg}$ BID and combination of DHA $150 \mathrm{mg}$ and Vitamin D 400 IU (OD) and the other arm received regular treatment. Children were analyzed at month 0 , month 3 and month 6 based on the Questionnaire.

Collection of Data: The questionnaire was created in reference to Ages and Stages Questionnaire (ASQ) [22, 23]. The main parameters studied are communication, gross motor, fine motor, problem solving, social and vocabulary skills. The approach adopted was pragmatic to ensure a robust and easy to use scale that can be used to obtain information from parents / careers of individuals with DS regardless of severity of ID, age, or comorbidities, and we therefore aimed to include children between $1-3$ years.

The child was observed carefully during each visit and the assessed for the each parameter with the questionnaire by the qualified trained assessors. During the initial visit questionnaire at Base line visit was administered by the study team and data was collected. The necessary administration details were provided to the parent. Participants were monitored throughout the study by telephone and details were collected using the questionnaire. At the end of the study (End of the study), final assessment was done using the questionnaire. 
Inclusion Criteria: The Children of age between 1-3 years of both sex with DS, confirmed by Karyotyping or genetic analysis with satisfactory health as determined by the Investigator on the basis of medical history and physical examination were included in the study. The child's legal parents, literate provided written informed consent prior to undergoing any procedures for all pertinent aspects of the study and were willing and able to comply with scheduled visits, provide proper answers for the questionnaires and telephonic follow up.

Exclusion Criteria: Children with Cardiac complications and any known laboratory abnormality which, in the opinion of the Investigator, would contraindicate study participation were excluded from the study. Children with active malignancies of any type, malignancies that had recurrence within 2 years before enrolment or basal cell carcinomas that have not been treated successfully were not included. Children with a history of clinically significant hypersensitivity to any drugs used to treat the underlying disease which has a cross sensitivity to the medications used in this study were excluded from the study. Children with hypothyroidism were also excluded.

Statistical Analysis: Chi square test was used to test our main outcome using SPSS version 17 software. Any statistical tests with $p$ - value of less than 0.05 was considered as statistically significant.

\section{Results}

Totally 60 children were participated in the study. Children were split in to two groups, one group ( $\mathrm{n}=30$ ) received the nutritional supplement of L-Carnosine, DHA and Vitamin D3. The other group of children $(n=30)$ were the control group who didn't receive any treatment. The gender ratio of 1:1 was maintained in both groups. Total duration of the study is 6 months. The outcomes of the study were analyzed for four major categories such as communication skills, Gross motor skills, fine motor, Problem solving and social skills.

Communication skills- The base line Visit (Month0), Month 3 andthe end of the study (Month 6) were compared, the children at the end of the study pointed the picture, imitated the two word sentence and followed instructions significantly $(\mathrm{p}<0.05)$ when compared to the Base line visit. The responders who said "no" for the particular activity at base line visit was significantly $(\mathrm{p}<0.05)$ improved upon treatment by responding "yes". On comparison with the cases and control at the end of the study, the children who were treated (cases) showed significant $(\mathrm{p}<0.05)$ improvement than the nontreated (control) children. Our study suggests that improvement in pointing the exact picture / object correctly in correlation to following upon the instructions justifies the improvement in cognition (Figure 1). This was not possible before the consumption of the supplement

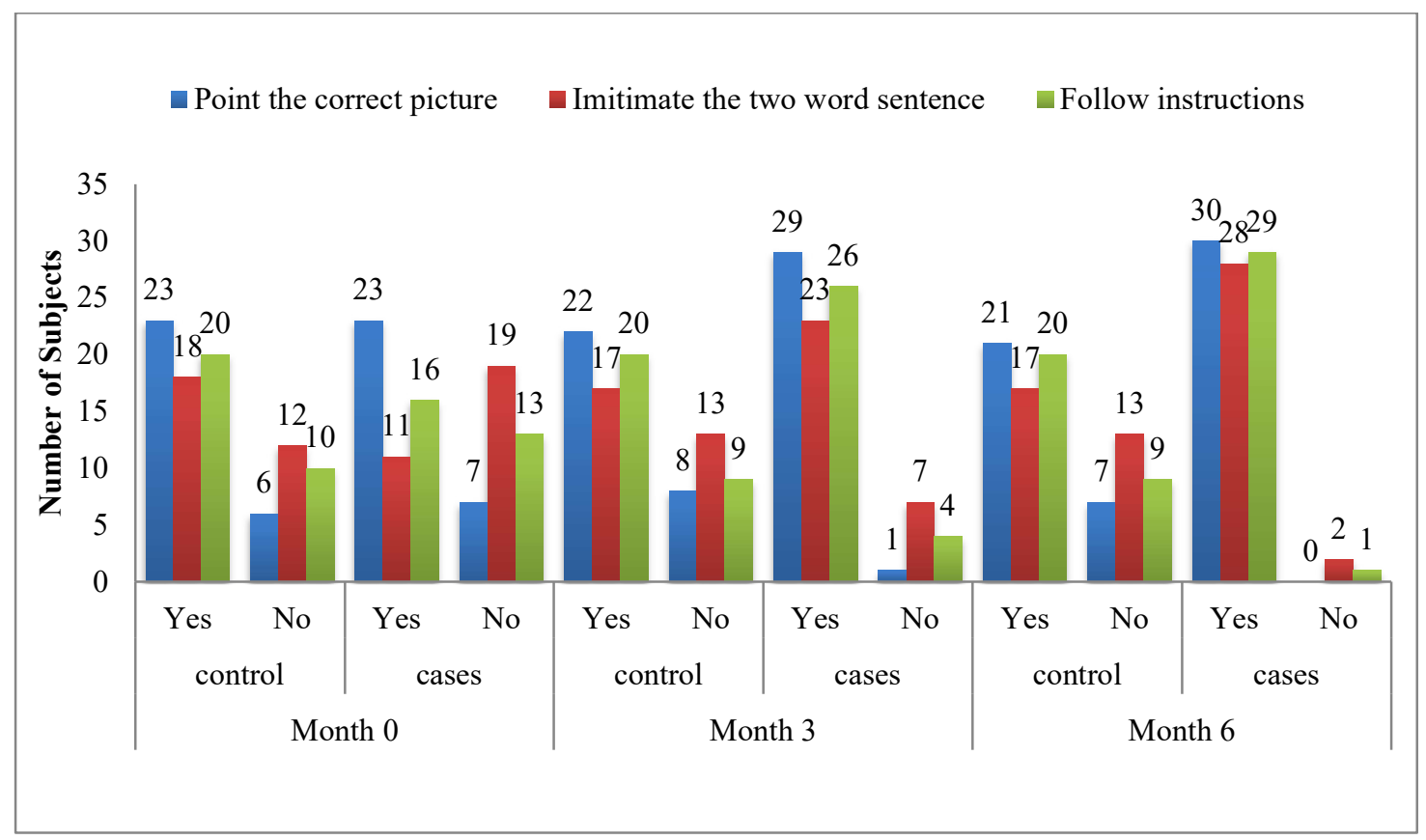

Figure-1: Communication skills: comparison between the control group and treatment group at month 0 , month 3 and month 6 
Gross Motor Skills: The base line Visit (Month0), Month 3 andthe end of the study (Month 6) were compared, the following activities like climbing, kicking and running had significantly $(\mathrm{p}<0.05)$ improved when compared to the Base line visit. The responders who said "no" for the particular activity at base line visit was significantly $(\mathrm{p}<0.05)$ improved upon treatment by responding "yes". On comparison with the cases and control at End of the study, the children who were treated (cases) improved in the following parameters like climbing, kicking and running significantly $(\mathrm{p}<0.05)$ when compared to the non treated (control) children (Figure 2).

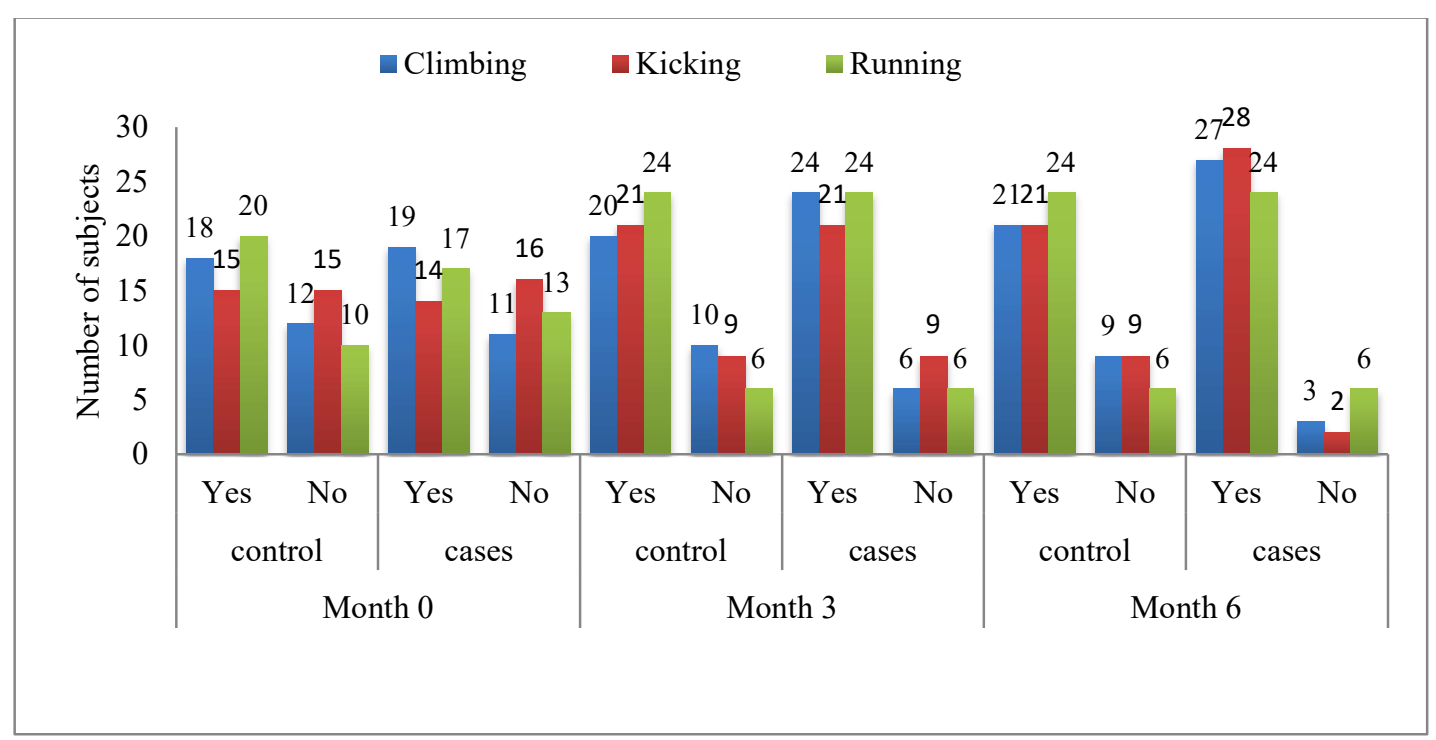

Figure- 2: Gross Motor skills: comparison between the

Control group and treatment group at month 0 , month 3 and month 6

Fine Motor- The children at theend of the study the following activities like eating food in a spoon, turning the page and turning motion had significantly $(\mathrm{p}<0.05)$ improved when compared to the Base line visit. The responders who said "no" for the particular activity at base line visit was significantly $(\mathrm{p}<0.05)$ improved upon treatment by responding "yes" when compared at End of the study (Figure 3). Following parameters like feeding, holding and opening bottles were also able to be performed, showing improvement significantly $(\mathrm{p}<0.05)$ when compared to thenon-treated (control) children.

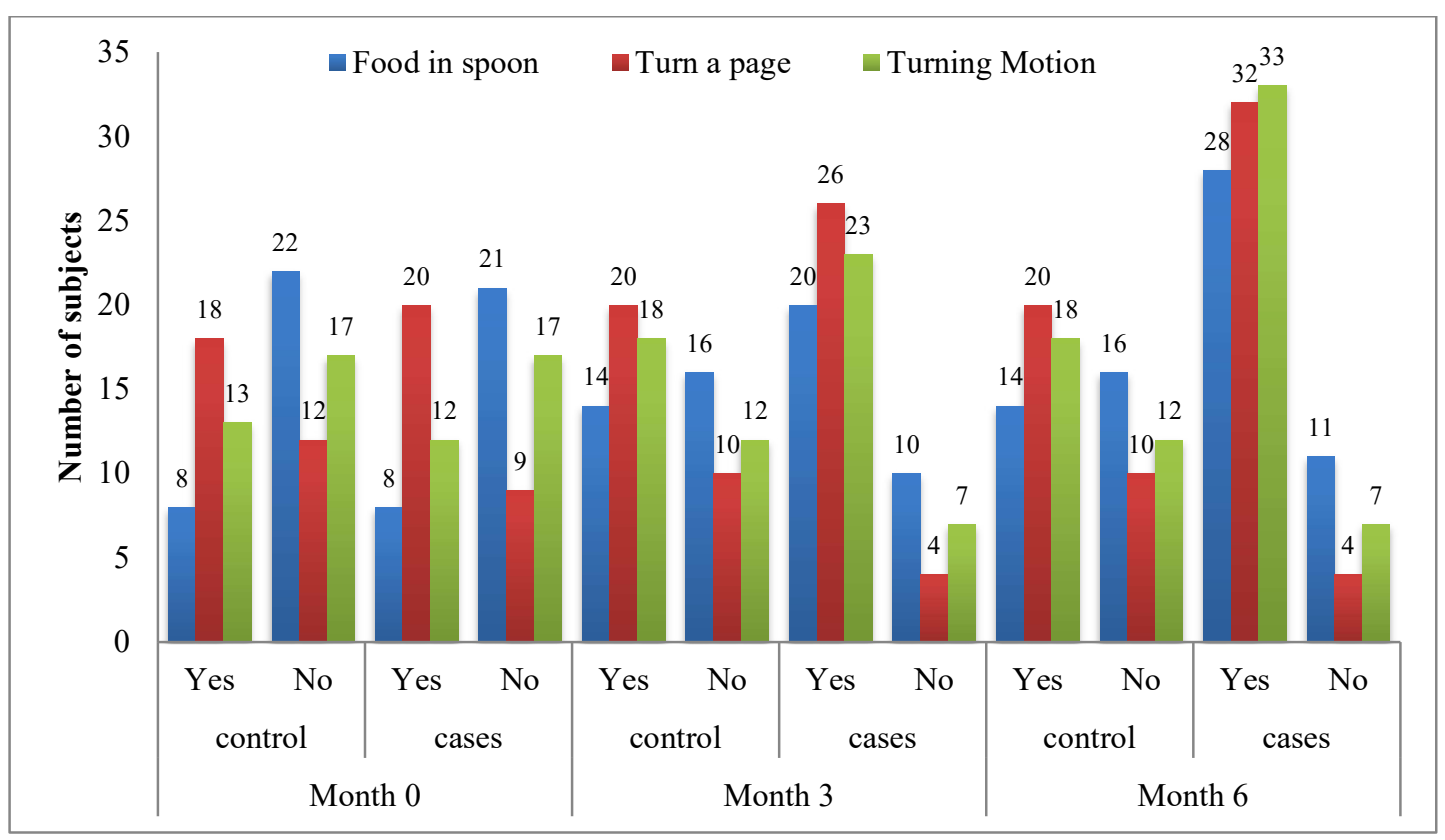

Figure 3: Fine Motor skills: comparison between the

control group and treatment group at month 0 , month 3 and month 6 
Problem solving: Activities like drawing a line, following actions and pretend play had significantly $(\mathrm{p}<0.05)$ improved in the supplemented children when compared to the base line visit and with control group. The responders who said "no" for the particular activity at base line visit had significantly $(\mathrm{p}<0.05)$ improved upon treatment by responding "yes" when compared at end of the study (Figure 4). On comparison with the cases and control at the end of the study, the children who were treated (cases) the following parameters like drawing a line, following actions and pretend play had significantly $(\mathrm{p}<0.05)$ improved when compared to the non treated (control) children.

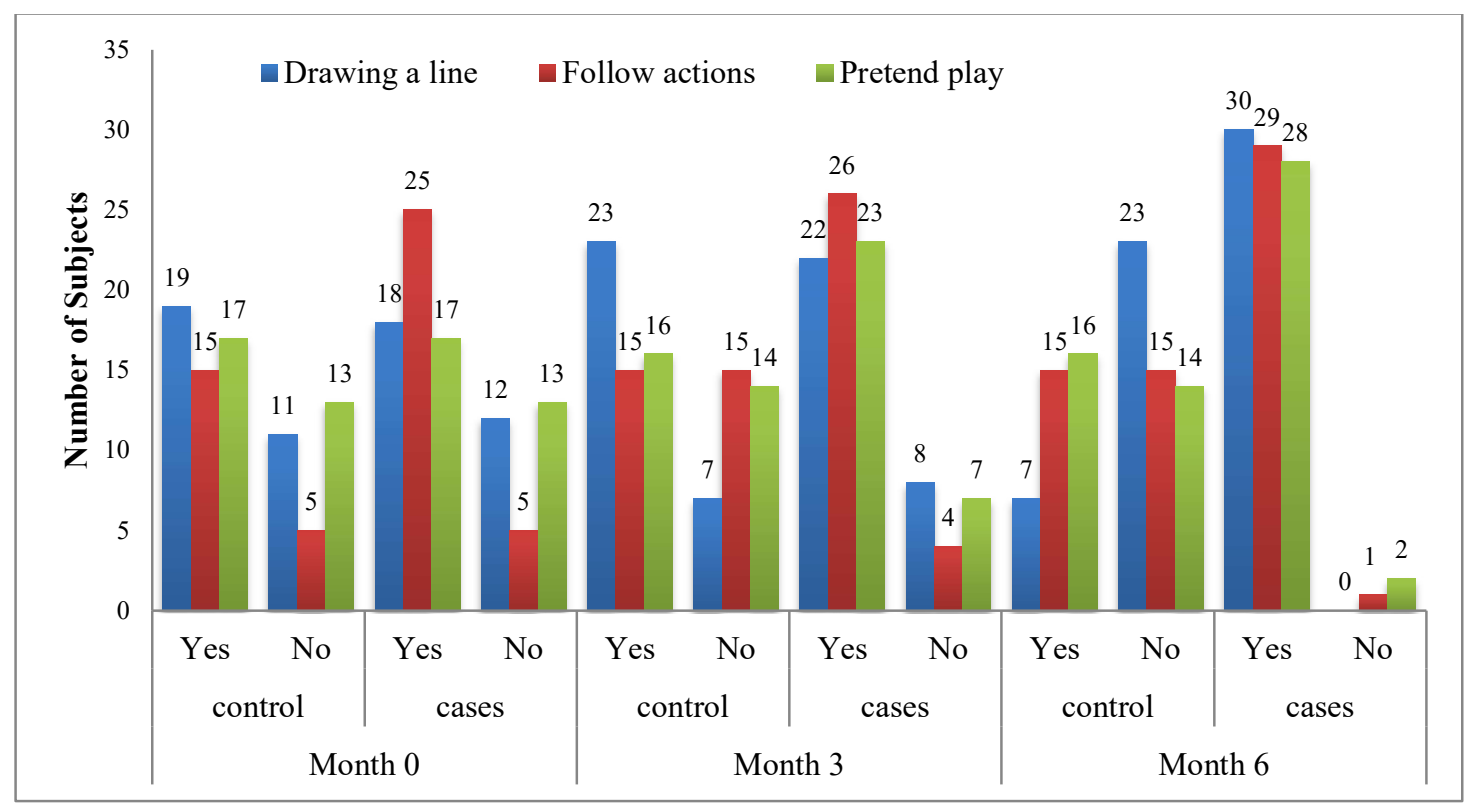

Figure-4: Problem solving: comparison between the control group and treatment group at month 0 , month 3 and month 6

Social skills: The children at the end of the study, the following activities like drinking without spilling, imitating activities and eating with fork had significantly $(\mathrm{p}<0.05)$ improved when compared to the base line visit. The responders who said "no" for the particular activity at base line visit had significantly $(p<0.05)$ improved upon treatment by responding "yes" when compared at the end of the study (Figure 5). On comparison with the cases and control at the end of the study, the children who were treated (cases), the following parameters like drinking without spill, imitating activities and eating with fork had significantly improved $(\mathrm{p}<0.05)$ when compared to the non-treated (control) children.

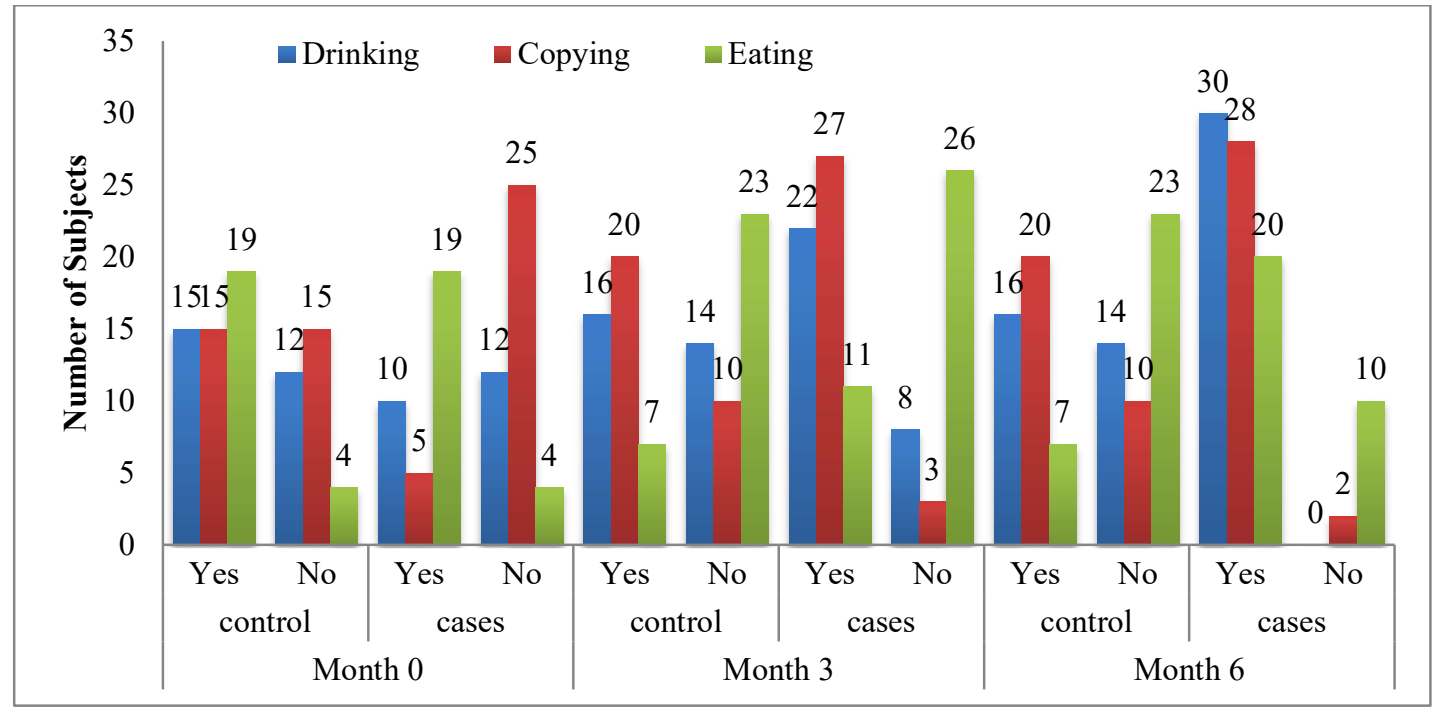

Figure-5: Social skills: comparison between the

control group and treatment group at month 0 , month 3 and month 6 
Vocabulary skills: On comparison with the month 0 vs 6 , the children at month 6 the following activities like calling the caregiver, identifying toys and communicating things had significantly $(\mathrm{p}<0.05)$ improved when compared to the month 0 . The responders who said "no" for the particular activity at month 0 had significantly $(\mathrm{p}<0.05)$ improved upon treatment by responding "yes" when compared at month 6 .Between the cases and control at month 3 , the children who were treated (cases), in the following parameters like calling the caregiver, identifying toys and communicate things showed significant improvement $(\mathrm{p}<0.05)$ when compared to the non-treated children (Figure 6 ).

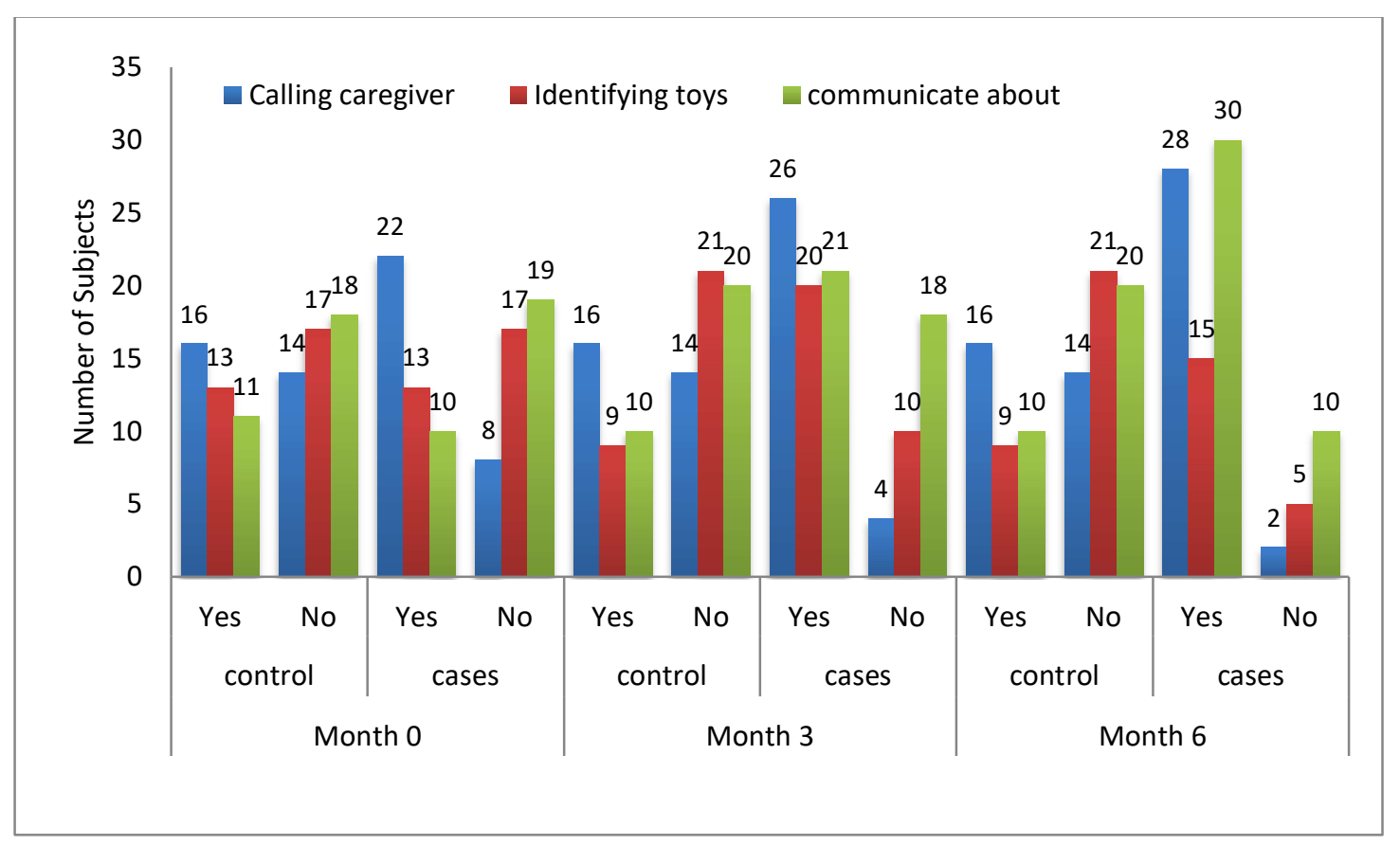

Figure-6: Vocabulary skills: comparison between the control group and treatment group at month 0 , month 3 and month 6

\section{Discussion}

Our results confirm that parameters related to communication, fine motor, gross motor, social skills, problem solving and vocabulary significantly improved in children who were supplemented with the nutritional supplement, when compared to the children who were not. Earlier studies of oral antioxidant drugs used to improve development in children with Down syndrome have included only a few participants and the results of these studies have not shown clear benefits of those drugs [24-26].

Research has revealed that many children with Down syndrome exhibit language delays, particularly in expressive abilities. Our study suggests that improvement in pointing to the exact picture / object correctly in correlation to following the instructions justifies the improvement in cognition. With L-Carnosine as well as increases in language comprehension that reached statistical significance. It have been proved that supplementation with carnosine significantly improved impressive and receptive speech, socialization, and behavior [12]. DHA and Vitamin D3 found to facilitate development of new synapses and to improve learning and memory abilities [13]. Thus this study also communicates to us that $\mathrm{L}$ Carnosine (CogniCare ${ }^{\mathrm{TM}}$ ) in support to DHA and Vitamin D might demonstrate improvement in communication in children with Down's syndrome. In children with Down syndrome, decreased muscle strength, low muscle tone, hyper mobility in joints defines the rate of gross motor development [27]. In our current study skills involving the three physical problems (climbing, running and jumping) improved upon consumption of the nutritional supplement. This might be due to replenishment of Carnosine levels in skeletal muscle, DHA and Vitamin D.

Fine motor skill efficiency significantly influences the quality of the task outcome as well as the speed of task performance. In this study feeding, holding hands and opening bottles had improved which showed that the stability, bilateral coordination had improved probably due to the nutritional supplement. Antioxidants have been reported to increase fine motor skills. 
Difficulties with problem solving persist in later development in this population and have been implicated in poorer task persistence and the increased levels of help-elicitation during task completion frequently observed in children with Down syndrome [28]. Activities like drawing a line, following actions and pretend play significantly improved on treatment in the children. Children with Down syndrome are able to use early nonverbal communication for social communication and sharing purposes (i.e., joint attention) and intention to regulate another's behaviour and complete tasks [29]. Activities like drinking without spill, imitating activities and eating with fork were significantly $(\mathrm{p}<0.05)$ improved on treatment.

Children with Down syndrome develop spoken language skills and learn vocabulary and grammar in the same order as other children. Similarly, studies have found the same links between spoken vocabulary size, combining words and developing grammar for children with Down syndrome as other children.[30] Supplementation of our nutrients improved vocabulary skills. They were able to communicate better to the caregiver which is seen in our results. Earlier pronunciation of single words, use of two word, two sentences, and identification of themselves by name showed significant improvement.

L-Carnosine supplementation yields an increase in cognitive processing speed, an increase in language skills, and improved motor skills. L Carnosine has a protective or activating role in the frontal lobe [3] DHA and Vitamin D3 were found to facilitate development of new synapses and to improve learning and memory abilities [13] and children who were supplemented with L-carnosine, DHA and Vitamin D3 showed improvement in various skills. When compared to the children who were not treated and the data were statistically significant $\mathrm{p}<0.05$

Contributors: Dr Priya Chandrasekhar and Dr Surekha Ramchandranhad planned, designed and guided the study. Dr Priya Chandrasekhar had collected the data, analysed it and prepared the manuscript.

What this study adds: Supplementation ofL-Carnosine along with DHA and Vitamin D3 yields an increase in cognitive processing speed, an increase in language skills, and improved motor skills.

\section{Conclusion}

This study confirms the effect of L-carnosine, DHA and vitamin D3 on children with down syndrome in improving parameters related to communication, fine motor, gross motor, social skills, problem solving and vocabulary significantly.

Acknowledgement: Authors thank Mr. Prabhu Daniel for his research assistance in carrying out this study.

Funding: Nil, Conflict of interest: None initiated, Perission from IRB: Yes

\section{References}

1. Lakhan, R. and M.T. Kishore, Down syndrome in tribal population in India: A field observation. Journal of neurosciences in rural practice, 2016. 7(1): p. 40.

2. Ralston, C. and R. Partridge, Early Communication Skills for Children with Down Syndrome: A Guide for Parents and Professionals. Journal of Developmental \& Behavioral Pediatrics, 2004. 25(5): p. 365-366.

3. Lott IT. Antioxidants in Down syndrome. Biochim Biophys Acta. 2012 May; 1822 (5):657-63. doi: 10. 1016/j.bbadis.2011.12.010. Epub 2011 Dec 21.

4. Muchová J, Žitňanová I, Ďuračková Z. Oxidative stress and Down syndrome. Do antioxidantsplay a role in therapy? Physiol Res. 2014;63(5):535-42. Epub 2014 Jun 5.

5.Kedziora J, Bartosz G. Down's syndrome: a pathology involving the lack of balance of reactive oxygen species. Free RadicBiol Med. 1988;4(5):317-30.

6. Hipkiss AR, Brownson C, Bertani MF, Ruiz E, Ferro A. Reaction of carnosine with aged proteins: another protective process? Ann N Y Acad Sci. 2002 Apr; 959: 285-94.

7. Boldyrev AA, Aldini G, Derave W. Physiology and pathophysiology of carnosine. Physiol Rev. 2013 Oct; 93 (4):1803-45. doi: 10.1152/physrev.00039.2012.

8. Young, W., Carnosine as a neuroprotective agent.

9. Chez, M.G., et al., Double-blind, placebo-controlled study of L-carnosine supplementation in children with autistic spectrum disorders. Journal of child neurology, 2002. 17(11): p. 833-837.

10. Bae, O.-N., et al., Safety and efficacy evaluation of carnosine, an endogenous neuroprotective agent for ischemic stroke. Stroke, 2013. 44(1): p. 205-212.

11. Hipkiss, A.R., et al., Carnosine: can understanding its actions on energy metabolism and protein homeostasis inform its therapeutic potential? Chemistry Central Journal, 2013. 7(1): p. 38. 
12. Sayre LM, Perry G, Smith MA. Oxidative stress and neurotoxicity. Chem Res Toxicol. 2008 Jan;21(1):17288. Epub 2007 Dec 4.

13. Gómez-Pinilla, F., Brain foods: the effects of nutrients on brain function. Nature Reviews Neuroscience, 2008. 9(7): p. 568.

14. Jiang LH, Shi Y, Wang LS, Yang ZR. The influence of orally administered docosahexaenoic acid on cognitiveability in agedmice. J Nutr Biochem. 2009 Sep; 20 (9): 735-41. doi: 10.1016/j.jnutbio.2008.07.003. Epub 2008 Sep 30.

15. Sakamoto, T., M. Cansev, and R.J. Wurtman, Oral supplementation with docosahexaenoic acid and uridine-5'-monophosphate increases dendritic spine density in adult gerbil hippocampus. Brain research, 2007. 1182: p. 50-59.

16. Sherwood NT, Lo DC. Long-term enhancement of central synaptic transmission by chronic brain-derived neurotrophic factor treatment. J Neurosci. 1999 Aug 15; 19 (16):7025-36.

17. Baum SJ, Kris-Etherton PM, Willett WC, Lichtenstein AH, Rudel LL, Maki KC, Whelan J, Ramsden CE, Block RC. Fatty acids in cardiovascular health and disease: a comprehensive update. J Clin Lipidol. 2012 May-Jun; 6 (3): 216-34. doi: 10.1016/j. jacl. 2012.04.077. Epub 2012 Apr 13.

18. Orme RP, Middleditch C, Waite L, Fricker RA. The Role of Vitamin $\mathrm{D}_{3}$ in the Development and Neuroprotection of Midbrain Dopamine Neurons. Vitam Horm. 2016; 100: 273-97.doi:10.1016/bs.vh. 2015. 10.007. Epub 2015 Dec 17.

19. Patrick RP, Ames BN. Vitamin D and the omega-3 fatty acidscontrolserotoninsynthesis and action, part2: relevance for ADHD, bipolar disorder, schizophrenia, and impulsive behavior. FASEB J. 2015 Jun;29(6): 2207-22. doi: 10.1096/fj.14-268342. Epub 2015 Feb 24.

20. Martinez, M., Developmental profiles of polyunsaturated fatty acids in the brain of normal infants and patients with peroxisomal diseases: severe deficiency of docosahexaenoic acid in Zellweger's and pseudo-Zellweger's syndromes, in Health Effects of
Omega 3 Polyunsaturated Fatty Acids in Seafoods. 1991, Karger Publishers. p. 87-102.

21. Hadley KB, Ryan AS, Forsyth S, Gautier S, Salem $\mathrm{N}$ Jr. The Essentiality of Arachidonic Acid in Infant Development. Nutrients. 2016 Apr 12; 8(4):216. doi: 10.3390/nu8040216.

22. Vaezghasemi, M., et al., Psychometric analysis of Age and Stages Questionnaire: Social-Emotional (ASQ: SE) among 3-year-oldsMasoud Vaezghasemi. European Journal of Public Health, 2017. 27(suppl_3).

23. Kvestad, I., et al., The assessment of developmental status using the Ages and Stages questionnaire-3 in nutritional research in north Indian young children. Nutrition journal, 2013. 12(1): p. 50.

24. Costa, A.C., On the promise of pharmacotherapies targeted at cognitive and neurodegenerative components of Down syndrome. Developmental neuroscience, 2011. 33 (5): p. 414-427.

25. Mohan, M., C. Bennett, and P.K. Carpenter, Memantine for dementia in people with Down syndrome. The Cochrane Library, 2009.

26. Lott IT, Doran E, Nguyen VQ, Tournay A, Head E, Gillen DL. Down syndrome and dementia: a randomized, controlled trial of antioxidant supplementation. Am J Med Genet A. 2011 Aug;155A (8): 1939-48.doi:10.1002/ajmg.a.34114.Epub2011 Jul 7.

27. Atkinson, S., et al., Intellectual disability in health and social care. 2014: Routledge.

28. Fidler, D.J., et al., Praxis skills in young children with Down syndrome, other developmental disabilities, and typically developing children. American Journal of Occupational Therapy, 2005. 59(2): p. 129-138.

29. Fidler, D.J., et al., Nonverbal requesting and problem-solving by toddlers with Down syndrome. American journal on mental retardation, 2005. 110(4): p. 312-322.

30. Martin GE, Klusek J, Estigarribia B, Roberts JE. LanguageCharacteristics of Individuals with Down Syndrome. Top Lang Disord.2009 Apr;29(2):112- 132.

How to cite this article?

Chandrasekhar P, Ramachandran S. Effect of L- Carnosine, DHA and vitamin D3 on children with down's syndrome- a pilot study on Indian population. Int J Pediatr Res. 2018;5(4):180-187. doi:10.17511/ijpr.2018.i04.05. 